\title{
ITERATIVE SOLUTION OF QUADRATIC TENSOR EQUATIONS FOR MUTUAL POLARISATION
}

\author{
WYNAND S. VERWOERD
}

Received 16 October 2001

\begin{abstract}
To describe mutual polarisation in bulk materials containing high polarisability molecules, local fields beyond the linear approximation need to be included. A second order tensor equation is formulated, and it describes this in the case of crystalline or at least locally ordered materials such as an idealised polymer. It is shown that this equation is solved by a set of recursion equations that relate the induced dipole moment, linear polarisability, and first hyperpolarisability in the material to the intrinsic values of the same properties of isolated molecules. From these, macroscopic susceptibility tensors up to second order can be calculated for the material.
\end{abstract}

2000 Mathematics Subject Classification: 35Q60, 74D10, 15A69, 81V55, 78A60.

1. Introduction. In developing polymer materials with the required optical properties needed for nonlinear optics technology, great advances have been made in recent years to design chromophore molecules with enhanced polarisabilities. For example, a highly successful strategy was developed by Marder et al. [1, 8, 9] based on a donoracceptor mechanism. By carefully matching the donor and acceptor strengths and the energetics of charge transfer, for example, by selecting acceptor structures that can compensate for the loss of aromaticity on polarisation in the intervening conductor part of the molecule, the hyperpolarisability of chromophores has been enhanced by orders of magnitude. For example, the simple prototype donor-acceptor molecule $p$ nitroaniline has a first hyperpolarisability [11] $\beta=6.3 \times 10^{-50}$ SI units at $\omega=1.17 \mathrm{eV}$, compared to the values [5] of $0.8 \times 10^{-50}$ and $0.4 \times 10^{-50}$, respectively, of its non-pushpull precursor molecules nitrobenzene and aniline. Moreover, fine tuning of the kind referred to has led to the production of several molecules with $\beta$ values [1,9] in a range as high as $400 \times 10^{-50}$ to $800 \times 10^{-50}$ SI units.

In view of these very high hyperpolarisabilities, it is appropriate to re-examine the approximations that are customarily used to calculate linear and nonlinear bulk susceptibilities from the corresponding molecular polarisabilities. This problem has a long history, having been studied by Lorentz [7] and a local field application to nonlinear optical properties introduced by Armstrong et al. [2], is commonly used and, for example, reviewed by Butcher and Cotter [3]. A key assumption in this treatment is that the local field experienced by a molecule is mainly due to the linear polarisability of surrounding molecules. As a result, the equation for the local field is effectively linearised and can be readily solved. However, with increasing nonlinear polarisabilities, this approximation must break down and it is of interest to solve the full nonlinear equation. 
Another notable property of donor-acceptor molecules is that their polarisabilities are highly anisotropic tensors. This is a direct result of the underlying mechanism, since the donor and acceptor groups are spatially localised, and it is mainly charge transfers along the geometrical axis that connect them, that is enhanced. This observation calls into question the adequacy of describing such molecules in terms of averaged scalar values, such as is typically measured by experiments on liquid suspensions or solutions. In particular, when the chromophore molecules are incorporated into a polymer, the structure can impose constraints on the orientation of chromophores; and at least some correlation between the orientation of a particular chromophore and its near neighbours that make significant contributions to the field it experiences, is to be expected. In the idealised case, chromophores can be modelled as located on a regular lattice with only a small number of discrete orientations allowed. The vector components of the local field, in this situation, can only be properly modelled by combining individual tensor components in a full tensor description of the mutual polarisation process.

The problem considered in this paper deals only with dipole moments interacting with the induced field. This is not necessarily the only physically important effectfor example, molecular quadropole moments interacting with field gradients may contribute substantially to the overall response of a polymer material to an optical stimulus. Similar problems about describing nonlinear response may be anticipated in that context, and the technique introduced here to deal with nonlinearity may also be of use for solving such problems.

The purpose of this paper is to present a method for solving a quadratic tensor equation for the dipole moment due to both an external field and mutual polarisation, and hence the effective polarisabilities in a bulk medium up to the first hyperpolarisability. No linearisation of the equations is done, and all tensor components are incorporated throughout. The method can be extended to higher order tensor equations and hyperpolarisabilities in a straightforward if tedious manner. From the effective polarisabilities, the corresponding susceptibilities can be calculated by simple summation.

The calculation of dipole interactions in molecular crystals has received extensive attention in the research literature as reviewed, for example, by Munn [10]. This paper concentrates on only one aspect of this broad field; namely, on finding a tensor solution to the nonlinear equation for induced polarisabilities. In this spirit, the next section gives only a brief account of the origin of this nonlinear equation; more details on the many ramifications of this subject can be found in the references. Section 3 presents the scalar solution in analytic form, both in order to establish relevant variables and to supply a basis of comparison with the iterative tensor solution that is the subject of Section 4 .

2. Problem formulation. The molecular polarisabilities are defined by the dipole moment $\dot{P}$ induced by the electric field $\dot{E}$ in which it is immersed

$$
\dot{P}=\dot{P}^{0}+\ddot{\alpha} \cdot \dot{E}+\frac{1}{2} \dddot{\beta}: \dot{E} \dot{E}
$$


Here tensors are decorated by dots to indicate their rank- $\dot{P}$ is a vector, $\ddot{\alpha}$ is a matrix or second rank tensor, and $\dddot{\beta}$ is a third rank tensor. The juxtaposition of vectors in the last term above indicates a dyadic product (in this case a matrix). The single dot product is the usual matrix product and the dyadic double dot product (:) is defined in tensor notation by

$$
(\dddot{A}: \ddot{B} \ddot{C})_{j k}^{i}=A_{m n}^{i} B_{j}^{n} C_{k}^{m},
$$

where the standard convention of summing over repeated tensor indices is assumed. In a bulk material, the local field $\dot{E}$ experienced by $\dot{P}$ is composed of the external field $\dot{E}^{0}$ and contributions $\dot{E}_{i}$ from its neighbours $\dot{P}_{i}$

$$
\dot{E}=\dot{E}^{0}+\sum \dot{E}_{i}
$$

In a liquid or other disordered system, substitution of (2.3) into (2.1) and expressing the $\dot{E}_{i}$ in terms of $\dot{P}_{i}$ lead to a set of coupled nonlinear equations for the $\dot{P}_{i}$. However, in a system, which is ordered at least locally over the spatial range of the dipole field, we can use the ordering to reduce it to a single equation. In a simple regular lattice formed, for example, by an idealised isotactic polymer, all dipoles are equal by symmetry. More generally, we assume that there may be more than one discrete set of dipoles, but that the moments in set $I$ are related to that of $\dot{P}$ by a relation $\dot{P}_{i}=\ddot{S}_{i} \cdot \dot{P}$, where $\ddot{S}_{i}$ is a fixed and known matrix that represents a symmetry operation (e.g., rotation or reflection with or without scaling). This could arise, for example, in a syndiotactic polymer where every second chromophore is rotated by a fixed angle.

The field experienced by the reference dipole $\dot{P}$ due to its neighbours $\dot{P}_{i}$, can hence be related to $\dot{P}$ itself by an expression for the dipole field factorised in terms of the Lorentz tensor $\ddot{L}$

$$
\dot{E}_{i}=f_{i} \ddot{L}_{i} \cdot \ddot{S}_{i} \cdot \dot{P} .
$$

Choosing the origin of the coordinate system at the position of $\dot{P}$ and using the standard expression for the dipole field, the factors $f$ and $\ddot{L}$ are given by explicit expressions of the form

$$
f_{i}=\frac{3}{4 \pi \varepsilon_{0} r_{i}^{3}}, \quad \ddot{L}_{i}=\left[\frac{\dot{r}_{i} \dot{r}_{i}}{r_{i}^{2}}-\frac{1}{3} \ddot{I}\right] .
$$

The Lorentz matrix $\ddot{L}_{i}$ determines the vector components of the field while the scalar factor $f_{i}$ carries the dependence on spatial separation. Since in a typical polymer the chromophore dimensions may be comparable to the dipole separation, use of the dipole approximation to calculate the field is not strictly justified and this may, to some extent, be compensated for by modifying the short distance behaviour of $f_{i}$, as investigated in a subsequent paper. Also, in extensions of this work, effects arising from gradients of the field may have to be included, though neglected here.

For the purpose of the derivation presented here, the detailed functional forms of the factors are unimportant, but it is noted that, by combining (2.3) and (2.4), the local field acting on $\dot{P}$ is determined by a field factor matrix $\ddot{F}$ defined by

$$
\sum \dot{E}_{i}=\left[\sum_{i} f_{i} \ddot{L}_{i} \cdot \ddot{S}_{i}\right] \cdot \dot{P} \equiv \ddot{F} \cdot \dot{P} .
$$


In a linear polymer chain or for a thin layer of such molecules, the convergence of the sum in the definition of the field factor matrix is not problematic since the dipole field drops off as $1 / r^{3}$ at large distances so that only near neighbours need to be included in the sum. In a bulk material, on the other hand, the sum formally has a logarithmic divergence and more sophisticated methods are needed to deal with this. This problem has been extensively studied, for example, in the work of Tomasi et al. [4] and the references contained therein, where the far field contribution is represented by introducing an appropriate surface charge distribution on the boundary of a cavity surrounding the molecule. In this paper, we are not concerned with the details of calculating the field factor but concentrate on a technique for calculating the induced dipole moment self-consistently once $\ddot{F}$ is known.

Substituting (2.6) into (2.1), the dipole moment $\dot{P}$, as a result of both an external field $\dot{E}^{0}$ and local field effects, is then to be found by solving the single quadratic tensor equation

$$
\dot{P}=\dot{P}^{0}+\left(\ddot{\alpha}^{0} \cdot \ddot{F}\right) \cdot \dot{P}+\frac{1}{2}\left(\dddot{\beta}^{0}: \ddot{F} \ddot{F}\right): \dot{P} \dot{P}+\dddot{\beta}^{0}:(\ddot{F} \cdot \dot{P}) \dot{E}^{0}+\ddot{\alpha}^{0} \cdot \dot{E}^{0}+\frac{1}{2} \dddot{\beta}^{0}: \dot{E}^{0} \dot{E}^{0} .
$$

Here, use has been made of the fact that $\dddot{\beta}$ is symmetric in its covariant indices, allowing interchange of the factors in the double dot product. The superscripts 0 have been added to the polarisability tensors to emphasise the fact that as with $\dot{P}^{0}$ they refer to the properties of the isolated molecule.

Once again, the calculation of these intrinsic hyperpolarisabilities is, for the purpose of this paper, considered to be a solved problem; it is by no means trivial, and a vast literature exists describing sophisticated methods such as the Time Dependent Hartree-Fock method (TDHF), proposed by Karna and Depuis [6]. However, our concern here is to solve (2.7) for the induced dipole moment $\dot{P}$ in the form of a power series-like tensor expression in the external field similar in form to (2.1), in such a way that the induced hyperpolarisabilities can be expressed in terms of known intrinsic molecular hyperpolarisabilities.

3. Scalar solution. It is instructive to first consider the solution of (2.7) in the scalar approximation where all tensor products become ordinary arithmetic products. In that case, (2.7) reduces to a quadratic equation in the scalar variable $P$ and its solution can be written down directly as follows:

$$
P=\frac{1}{\beta^{0} F^{2}}\left[1-\alpha^{0} F-\beta^{0} F E^{0}+\sqrt{1-2 \alpha^{0} F-2 \beta^{0} F E^{0}+\alpha^{02} F^{2}-2 \beta^{0} F^{2} P^{0}}\right] .
$$

The appropriate sign for the square root has been selected to obtain the correct form in the limit $\beta^{0} \rightarrow 0$, where (2.7) becomes linear, namely,

$$
P \rightarrow \frac{P^{0}+\alpha^{0} E^{0}}{1-\alpha^{0} F}
$$

which is the well-known result for linear response, so that the effect of the local field is a mutual depolarisation factor $1 /\left(1-\alpha^{0} F\right)$ that modifies both the intrinsic dipole moment and the linear polarisability. To extend this to the nonlinear case, we expand 
the root expression in (3.1) as a power series in $E^{0}$, keeping terms to second order. This leads naturally to the definition of depolarisation factors defined as $d=P / P^{0}$, $a=\alpha / \alpha^{0}$, and $b=\beta / \beta^{0}$ for the dipole moment, polarisability, and hyperpolarisability, respectively, that is, (3.1) expands into the form

$$
P=d P^{0}+a \alpha^{0} E^{0}+\frac{1}{2} b \beta^{0} E^{0^{2}} .
$$

The expressions for the depolarisation factors are reduced to a simple form by the introduction of the dimensionless variables

$$
x_{0}=\alpha^{0} F ; \quad y_{0}=\beta^{0} F^{2} P^{0}
$$

in terms of which the following expressions are obtained:

$$
\begin{aligned}
& d=\frac{1-x_{0}-\sqrt{1-2 x_{0}+x_{0}^{2}-2 y_{0}}}{y_{0}}, \\
& a=\frac{1}{x_{0}}\left[\frac{1}{\sqrt{1-2 x_{0}+x_{0}^{2}-2 y_{0}}}-1\right], \\
& b=\sqrt{\left(1-2 x_{0}+x_{0}^{2}-2 y_{0}\right)^{3}} .
\end{aligned}
$$

Using plausible values for the free molecular polarisabilities, dipole magnitudes and separations, it is found that $x_{0}$ is a fraction of order 1 but $y_{0} \ll x_{0}$. For future reference, it is noted that (3.5) can be expanded as power series in the factor $y_{0} /\left(1-x_{0}\right)^{2}$ as follows:

$$
\begin{aligned}
& d=\frac{1}{1-x_{0}}+\frac{y_{0}}{2\left(1-x_{0}\right)^{3}}+\frac{y_{0}^{2}}{2\left(1-x_{0}\right)^{5}}+\frac{5 y_{0}^{3}}{8\left(1-x_{0}\right)^{7}}+\frac{7 y_{0}^{4}}{8\left(1-x_{0}\right)^{9}}+\cdots, \\
& a=\frac{1}{1-x_{0}}+\frac{y_{0}}{x_{0}\left(1-x_{0}\right)^{3}}+\frac{3 y_{0}^{2}}{2 x_{0}\left(1-x_{0}\right)^{5}}+\frac{5 y_{0}^{3}}{2 x_{0}\left(1-x_{0}\right)^{7}}+\frac{35 y_{0}^{4}}{8 x_{0}\left(1-x_{0}\right)^{9}}+\cdots, \\
& b=\frac{1}{\left(1-x_{0}\right)^{3}}+\frac{3 y_{0}}{\left(1-x_{0}\right)^{5}}+\frac{15 y_{0}^{2}}{2\left(1-x_{0}\right)^{7}}+\frac{35 y_{0}^{3}}{2\left(1-x_{0}\right)^{9}}+\cdots .
\end{aligned}
$$

Note that in the limit as $y_{0} \rightarrow 0$, the depolarisation factors reduce to powers of (1$\left.x_{0}\right)^{-1}$. This is the scalar simplification of the expression usually referred to [3] as the "local field factor," and the result above shows that the conventional proportionality of effective polarisabilities in a medium to powers of the local field factor only holds when $y_{0}$ can be neglected. Putting $y_{0}=0$ amounts to the linear simplification referred to in Section 1, that is, when evaluating the local field due to the neighbours from (2.3) and (2.4), only the linear term from (2.1) is included in (2.4), although, for the subsequent substitution of (2.3) into (2.1), the quadratic term in (2.1) is retained. The treatment presented above includes quadratic terms consistently at both stages and this leads to the additional terms in (3.6). 
4. Solution of the tensor equation. The scalar solution has shown that it is both important and simple to treat the first term that is linear in $\dot{P}$ on the right-hand side of (2.7) exactly, as this leads to the ubiquitous factor $\left(1-x_{0}\right)^{-1}$. We change (2.7) into a recursive form that incorporates this idea

$$
\begin{aligned}
\dot{P}_{n}=\left(1-\ddot{\alpha}^{0} \cdot \ddot{F}\right)^{-1} \cdot & {\left[\dot{P}^{0}+\frac{1}{2}\left(\dddot{\beta}^{0}: \ddot{F} \ddot{F}\right): \dot{P}_{n-1} \dot{P}_{n-1}\right.} \\
& \left.+\dddot{\beta}^{0}:\left(\ddot{F} \cdot \dot{P}_{n-1}\right) \dot{E}^{0}+\ddot{\alpha}^{0} \cdot \dot{E}^{0}+\frac{1}{2} \dddot{\beta}^{0}: \dot{E}^{0} \dot{E}^{0}\right] .
\end{aligned}
$$

The subscript $n$ indicates the order of the recursion, which is started by defining the zeroth-order recursion as $\dot{P}_{0} \equiv 0$. Further progress is facilitated by introducing a number of dimensionless vectors and tensors in analogy to the variables in the scalar analysis.

Equation (3.4) shows that the magnitude $P^{0}$ of the intrinsic molecular dipole moment plays the role of a scaling constant. In order to treat the case of molecules with zero intrinsic moment also using the same formalism, the scaling constant is reassigned by writing

$$
\dot{P}^{0}=P^{0} \dot{p}^{0} ; \quad \dot{p}^{0}= \begin{cases}\frac{\dot{P}^{0}}{\left|\dot{P}^{0}\right|}, & \text { if }\left|\dot{P}^{0}\right| \neq 0 \\ \dot{0}, & \text { otherwise. }\end{cases}
$$

While keeping $P^{0}$ as the magnitude of the moment of the isolated molecule for intrinsically polarised molecules, for unpolarised molecules any convenient value may be chosen, for example, such that the external field is scaled to a dimensionless vector of order unity by the following transformation:

$$
\dot{e}=\frac{1}{P^{0}} \ddot{F}^{-1} \cdot \dot{E}^{0}
$$

The variables $x$ and $y$ of the scalar treatment now become dimensionless tensors

$$
\ddot{x}_{0}=\ddot{\alpha}^{0} \cdot \ddot{F}, \quad \dddot{y}_{0}=P^{0} \dddot{\beta}^{0}: \ddot{F} \ddot{F} .
$$

In terms of these, the defining relation for intrinsic molecular polarisabilities, (2.1), can be written as

$$
\dot{p} \equiv \frac{\dot{P}}{P^{0}}=\dot{p}^{0}+\ddot{x}_{0} \cdot \dot{e}+\frac{1}{2} \dddot{y}_{0}: \dot{e} \dot{e} .
$$

To prove the equivalence, note that by the associative law of matrix multiplication

$$
\ddot{x}_{0} \cdot \dot{e}=\left(\ddot{\alpha}^{0} \cdot \ddot{F}\right) \cdot \frac{1}{P^{0}}\left(\ddot{F}^{-1} \cdot \dot{E}^{0}\right)=\frac{1}{P^{0}} \ddot{\alpha}^{0} \cdot \dot{E}^{0},
$$

and in a similar way, we have

$$
\dddot{y}_{0}: \dot{e} \dot{e}=\left(P^{0} \dddot{\beta}^{0}: \ddot{F} \ddot{F}\right): \frac{1}{\left(P^{0}\right)^{2}}\left(\ddot{F}^{-1} \cdot \dot{E}^{0}\right)\left(\ddot{F}^{-1} \cdot \dot{E}^{0}\right)=\frac{1}{P^{0}} \dddot{\beta}^{0}: \dot{E}^{0} \dot{E}^{0},
$$


where the last step now follows from the following identity for the double dot product that can be proven by the application of the tensor component expansion of (2.2)

$$
(\dddot{A}: \ddot{B} \ddot{D}): \ddot{C} \ddot{E}=\dddot{A}:(\ddot{B} \cdot \ddot{C})(\ddot{D} \cdot \ddot{E}) .
$$

By similar tensor manipulations, (4.1) is transformed into

$$
\dot{p}_{n}=\ddot{D} \cdot\left[\dot{p}^{0}+\ddot{x}_{0} \cdot \dot{e}+\frac{1}{2} \dddot{y}_{0}: \dot{e} \dot{e}+\dddot{y}_{0}: \dot{p}_{n-1} \dot{e}+\frac{1}{2} \dddot{y}_{0}: \dot{p}_{n-1} \dot{p}_{n-1}\right]
$$

where, for convenience, the definition of the dimensionless matrix $\ddot{D} \equiv\left(1-\ddot{x}_{0}\right)^{-1}$ was introduced.

Conceptually, what needs to be done is to solve (4.9) for the vector $\dot{p}_{n}$ as a function of $\dot{e}$, and subsequently expand this as a power series in $\dot{e}$ in order that the various hyperpolarisabilities can be identified as coefficients (the process that was explicitly performed in the scalar case). Considering the form of the scalar solution in (3.1), the difficulty in doing that would first of all be to attach some meaning to the concept of the square root of a tensor expression. Moreover, (4.9) contains 3 different kinds of tensor products-the dyadic, dot, and double dot-which obscure the sense in which it is a quadratic equation; and finally, in the envisaged series expansion, we may anticipate that once more a combination of the different kinds of tensor product will be involved, which is bound to make the solution vastly more complicated than a scalar power series.

The solution presented here cuts across these difficulties by using algebraic iteration to derive a set of recursion formulas, from which the tensor power series can be constructed. We start with considering the desired end. If this result can be expressed in the same functional form as (4.5); namely,

$$
\dot{p}_{n}=\dot{w}_{n}+\ddot{x}_{n} \cdot \dot{e}+\frac{1}{2} \dddot{y}_{n}: \dot{e} \dot{e}
$$

the coefficients in (4.10) would clearly represent the effective polarisabilities in the medium. The first iteration of (4.9), using $\dot{p}_{0}=\dot{0}$, is already in this form and allows the identifications

$$
\dot{w}_{1}=\ddot{D} \cdot \dot{p}^{0} ; \quad \ddot{x}_{1}=\ddot{D} \cdot \ddot{x}_{0} ; \quad \dddot{y}_{1}=\ddot{D} \cdot \dddot{y}_{0}
$$

Using (4.10) and (4.11), we can simplify (4.9) to read

$$
\dot{p}_{n}=\dot{p}_{1}+\frac{1}{2} \dddot{y}_{1}:\left[2 \dot{p}_{n-1} \dot{e}+\dot{p}_{n-1} \dot{p}_{n-1}\right]
$$

To obtain the solution of (4.12) in the desired functional form, $\dot{p}_{n}$ on the left- and $\dot{p}_{n-1}$ on the right-hand side are both substituted from (4.10). In order to identify the effective hyperpolarisabilities, the resulting expression should revert to the same 
tensor structure as (4.10). However, inspection of these equations shows that the result of the substitution has a far more complicated tensor product structure. For example, some of its terms contain two individual factors $\dot{e}$ in combination with other tensors, whereas the form of (4.10) requires that the only form that is quadratic in $\dot{e}$ should be the electric field dyadic. Nevertheless, we have succeeded in transforming all terms to a form consistent with (4.10) by use of the following set of identities for the double dot product that may be derived by using (2.2) and analogous component expansions

$$
\begin{gathered}
\dddot{A}: \dot{B} \dot{C} \equiv(\dddot{A} \cdot \dot{B}) \cdot \dot{C}, \\
\dddot{A}: \dot{B} \dddot{C} \equiv(\dddot{A} \cdot \dot{B}) \cdot \dddot{C}, \\
\dddot{A}: \dot{B}(\ddot{C} \cdot \dot{D}) \equiv(\dddot{A}: \dot{B} \ddot{C}) \cdot \dot{D}, \\
\dddot{A}:(\ddot{B} \cdot \dot{C}) \dot{D} \equiv(\dddot{A} \cdot \ddot{B}): \dot{C} \dot{D}, \\
\dddot{A}: \dot{B}(\dddot{C}: \dot{D} \dot{E}) \equiv(\dddot{A}: \dot{B} \dddot{C}): \dot{D} \dot{E} \equiv[(\dddot{A} \cdot \dot{B}) \cdot \dddot{C}]: \dot{D} \dot{E} .
\end{gathered}
$$

Comparing coefficients of each order in $\dot{e}$ in the rearranged version of (4.12), the following set of recursion relations is obtained in orders 0,1 , and 2 , respectively:

$$
\begin{gathered}
\dot{w}_{n}=\dot{w}_{1}+\frac{1}{2} \dddot{y}_{1}: \dot{w}_{n-1} \dot{w}_{n-1}, \\
\ddot{x}_{n}=\ddot{x}_{1}+\dddot{y}_{1} \cdot \dot{w}_{n-1}+\dddot{y}_{1}: \dot{w}_{n-1} \ddot{x}_{n-1}, \\
\dddot{y}_{n}=\dddot{y}_{1}+2 \dddot{y}_{1} \cdot \ddot{x}_{n-1}+\dddot{y}_{1}: \ddot{x}_{n-1} \ddot{x}_{n-1}+\left(\dddot{y}_{1} \cdot \dot{w}_{n-1}\right) \cdot \dddot{y}_{n-1} .
\end{gathered}
$$

The rearrangements that were needed to express the $\dot{e}$ factors appropriately dictate, at the same time, the combinations of tensor products in (4.14), (4.15), and (4.16), and as shown below, this carries through to the tensor equivalent of the scalar power series (3.6). In this way, one of the problems mentioned in the discussion of (4.9) is addressed very straightforwardly.

Equations (4.14), (4.15), and (4.16), together with the definitions (4.2), (4.4), and (4.11), form the key set of equations that allows us to proceed directly from a known set of intrinsic molecular polarisabilities as contained in $\dot{P}^{0}, \ddot{\alpha}^{0}$, and $\dddot{\beta}^{0}$, to the induced polarisabilities for the bulk medium in an external field that is embodied in $\dot{w}_{n}, \ddot{x}_{n}$, and $\dddot{y}_{n}$. The latter are, by construction, iterative approximations to the underlying material properties for which the obvious notation is $\dot{w}, \ddot{x}, \dddot{y}$. The recursion has a hierarchical structure, first equation (4.14) can be iterated to the desired degree of accuracy, then the results of this are used to iterate (4.15), and, in turn, the results from both of these are needed to iterate (4.16).

To regain the physical polarisation tensors from the dimensionless forms, $\dot{e}$ is substituted from (4.3) into (4.10), and by comparison with (2.1), the induced or effective polarisabilities are given by

$$
\begin{gathered}
\dot{P}^{i}=P^{0} \dot{w}, \\
\ddot{\alpha}=\ddot{x} \cdot \ddot{F}^{-1}, \\
\dddot{\beta}=\frac{1}{P^{0}} \dddot{y}: \ddot{F}^{-1} \ddot{F}^{-1},
\end{gathered}
$$


where $P^{i}$ represents the modified intrinsic moment as a result of local fields, but in the absence of an external field.

The claim that the iterative solution presented here is equivalent to an analytical solution, can be confirmed by noticing that when (4.17), (4.18), and (4.19) are reduced to scalar equivalents, they imply the following relationships to the depolarisation factors introduced in Section 3:

$$
d=w ; \quad a \equiv \frac{\alpha}{\alpha^{0}}=\frac{x}{x_{0}} ; \quad b \equiv \frac{\beta}{\beta^{0}}=\frac{y}{y_{0}} .
$$

When the scalar equivalents of the tensor recursions in (4.14), (4.15), and (4.16) are performed algebraically by repeated substitution, it is easily seen that power series, which are identical to those of (3.6), are generated.

Moreover, this comparison gives information about the convergence of the iteration. It is observed that in the $n$th iteration of (4.14) and (4.15), all terms up to the $(n-1)$ th order in $y_{0} /\left(1-x_{0}\right)^{2}$ are correct, while, in the case of (4.16), correct terms up to order $(n-2)$ are obtained. As this observation reflects the numerical coefficients in the expansion, it can also be transferred to the case of the tensor recursion to give a guideline for truncating the iteration.

The iterative solution has an important advantage over the analytical one in that it applies also to higher order optical constants. For example, if the second order hyperpolarisability term is also included in (2.1), even for the scalar case, the calculation of induced optical constants from the resulting cubic equation becomes very complicated. The extension of the iterative solution to this case is, by contrast, straightforward, although tedious in performing the tensor algebra corresponding to (4.13).

Returning to the case of the quadratic tensor equation, it is noted that the concept of a depolarisation factor is not useful beyond the linear polarisation term in this case. The reason is demonstrated by $(4.20)$, while $\ddot{x}_{0}$ is still a matrix and can be inverted to define a depolarisation matrix $\ddot{a}, \dddot{y}_{0}$ is a third rank tensor and cannot be inverted to produce a tensor equivalent of $b$. However, this is of no consequence since (4.19) gives a well-defined prescription for finding the induced hyperpolarisability.

In principle, also for the tensor case, the recursions in (4.14), (4.15), and (4.16) can be done algebraically by repeated substitution. In fact, this generates a power series with the required mix of tensor products as a direct consequence of the manipulations that were needed to derive those equations. However, in order to avoid the accumulation of involved tensor products, it was found more practical to apply the equations numerically. Since the result of the tensor products on the right-hand side always return a tensor of the appropriate rank as specified by the left-hand side, subsequent iterations do not increase in complexity provided that the results of previous iterations are stored for reuse.

Finally, we consider the calculation of macroscopic susceptibilities. Substituting back the solutions of (4.14), (4.15), and (4.16) into (4.10) gives an expression for the induced molecular dipole moment, which depends explicitly only on the external field, and can be summed over all dipoles in a suitably chosen unit cell to give the polarisation of the medium. Assuming that the external field is homogeneous on a molecular 
scale, it can be factored out of the sums on the right-hand side, and using the conventional factors in the definition [3] of the $n$th order susceptibility tensor $\chi^{n}$, we get

$$
\chi^{n}=\alpha^{n}{ }_{c} / n ! \varepsilon_{0} V
$$

Here, $V$ is the volume of the unit cell, which is chosen large enough to contain a representative collection of molecular orientations, and the cellular total of the $n$th polarisability is given by

$$
\begin{aligned}
& \alpha_{c}^{1}=\ddot{\alpha}+\sum_{j} \ddot{S}_{j} \cdot \ddot{\alpha} \cdot \ddot{S}_{j}^{-1}, \\
& \alpha_{c}^{2}=\dddot{\beta}+\sum_{j} \ddot{S}_{j} \cdot \dddot{\beta}: \ddot{S}_{j}^{-1} \ddot{S}_{j}^{-1} .
\end{aligned}
$$

The first term in each of the equations in (4.22) refers to the polarisability of the molecule or chromophore at the origin that defines the coordinate system in which the tensors are determined, and the sum over $j$ extends over all other chromophores in the unit cell. Note that for chromophore $j$, its polarisability is given by an equation like (2.1), but in its own coordinate system, that is, the effective external field it experiences is $S_{j}^{-1} \cdot \dot{E}$. From this consideration, the $S$-factors in (4.22) are derived.

5. Conclusions. The conceptual framework for this study was the case of identical highly polarisable and anisotropic molecular chromophore molecules embedded in a regular lattice formed, for example, by a polymer backbone. In this case, it was shown that mutual polarisation of the chromophores, with or without an external electric field, is determined by a single tensor equation, which is nonlinear in the molecular dipole moment. The equation includes nonlinearity in the external field as well as nonlinearity in the local field in terms of higher order polarisabilities. By rewriting this equation in a recursive form, it was shown that it can be solved iteratively in a way that directly expresses the induced (effective) polarisability tensors of a molecule as a result of both the external field and the mutual interactions in terms of the intrinsic polarisabilities of an isolated molecule. From this, the nonlinear susceptibility tensors of the bulk medium can be calculated in a straightforward way. No tensor averaging is used, so that the interaction between different tensor components of neighbouring molecules is fully included.

The idea of using iteration to solve such a nonlinear equation has also been pursued by other authors, for example, in the work of Munn [10] where a nonlinear equation for the local field is solved in this manner. However, the advantage of the present formalism is that by eliminating the explicit appearance of the local field, the induced polarisabilities are directly expressed in terms of the intrinsic polarisabilities. Moreover, this is done by a set of recursion relations, which are in themselves exact solutions of the nonlinear equation (as demonstrated by the fact that they give rise to the same infinite power series as the analytical solution, for the scalar case). Although iteration of the recursion relations also needs to be truncated in practice, the truncation order can be decided purely on the grounds of the intrinsic polarisabilities and is not related to truncation of the expansions in the orders of the electric field. 
The derivation presented only included terms up to the first hyperpolarisability, that is, second order susceptibility. However, the method used allows a straightforward extension to higher order nonlinearity.

Representing an optical polymer as a regular lattice is certainly a highly idealised abstraction. In most of the derivation, however, only limited use was made of this assumption. To reduce the problem from coupled equations in a disordered material to a single equation as in (2.7), it is only necessary to have a local ordering where the neighbours (within range of the dipole field) of a given chromophore have fixed (but not necessarily identical) orientations and positions. Under this much more realistic assumption, the resulting equation is solved essentially exactly, depending on the number of iterations performed. Only at the very last step of finding bulk properties, was the concept of a repetitive unit cell invoked, implying a long range ordering as well. This assumption could be relaxed without affecting the rest of the derivation by assuming such unit cells to be uncorrelated and doing a statistical average over different unit cell orientations. This idea is left for future work as the present focus is on describing the local effects as accurately as possible.

Finally, it is remarked that the equations presented are quite practical for numerical calculations, with all matrices and tensors in 3-space so that relatively small numbers of components are involved. This is to be further demonstrated in a subsequent paper where a practical application to calculations for specific chromophore molecules of the type mentioned in Section 1 is presented.

ACKNOWLedgments. The hospitality of the Physics Department at the University of Otago, Dunedin, New Zealand, where this project was initiated is gratefully acknowledged, as well as that of the Physics Department at the University of South Africa where the work was further developed.

\section{REFERENCES}

[1] M. Ahlheim, M. Barzoukas, P. V. Bedworth, M. Blanchard-Desce, A. Fort, Z.-Y. Hu, S. R. Marder, J. W. Perry, C. Runser, M. Staehelin, and B. Zysset, Chromophores with strong heterocyclic acceptors: a poled polymer with a large electro-optic coefficient, Science 271 (1996), 335-337.

[2] J. A. Armstrong, J. A. Bloembergen, J. Ducuing, and P. S. Pershan, Interactions between light waves in a nonlinear dielectric, Phys. Rev. 127 (1962), 1918-1939.

[3] P. N. Butcher and D. Cotter, The Elements of Nonlinear Optics, Cambridge Studies in Modern Optics, vol. 9, Cambridge University Press, Cambridge, 1990.

[4] R. Cammi, B. Mennucci, and J. Tomasi, On the calculation of local field factors for microscopic static hyperpolarizabilities of molecules in solution with the aid of quantummechanical methods, J. Phys. Chem. A 102 (1998), 870-875.

[5] V. J. Docherty, D. Pugh, and J. O. Morley, Calculation of the second-order electronic polarizabilities of some organic molecules, J. Chem. Soc. Faraday Trans. 81 (1985), 1179-1192.

[6] S. P. Karna and M. Dupuis, Frequency dependent nonlinear optical properties of molecules, J. Comput. Chem. 12 (1991), 487-504.

[7] H. A. Lorentz, The Theory of Electrons, Teubner, Leipzig, 1916, reprinted by Dover, New York, 1951.

[8] S. R. Marder, D. N. Beratan, and L.-T. Cheng, Approaches for optimising the first electronic hyperpolarizability of conjugated organic molecules, Science 252 (1991), 103-106. 
[9] S. R. Marder, L.-T. Cheng, B. G. Tiemann, A. C. Friedli, M. Blanchard-Desce, J. W. Perry, and J. Skindhoj, Large first hyperpolarizabilities in push-pull polyenes by tuning of the bond length alternation and aromaticity, Science 263 (1994), 511-514.

[10] R. W. Munn, Electric dipole interactions in molecular crystals, Mol. Phys. 64 (1988), 1-20.

[11] C. C. Teng and A. F. Garito, Dispersion of the nonlinear second-order optical susceptibility of organic systems, Phys. Rev. 28 (1983), 6766-6773.

Wynand S. Verwoerd: Centre for Advanced Computational Solutions, AMAC DiviSION, P.O. BOX 84, LINCOLN UNIVERSITY, CANTERBURY, NEW ZEALAND

E-mail address: verwoerw@incoln.ac.nz 


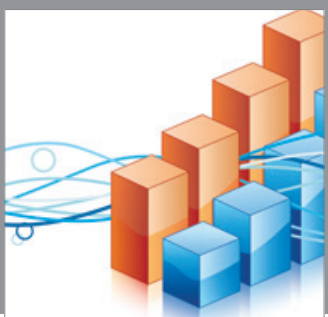

Advances in

Operations Research

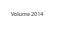

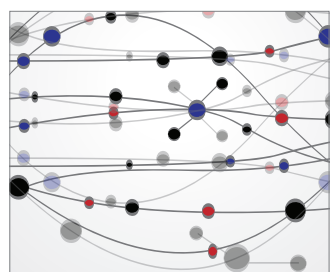

\section{The Scientific} World Journal
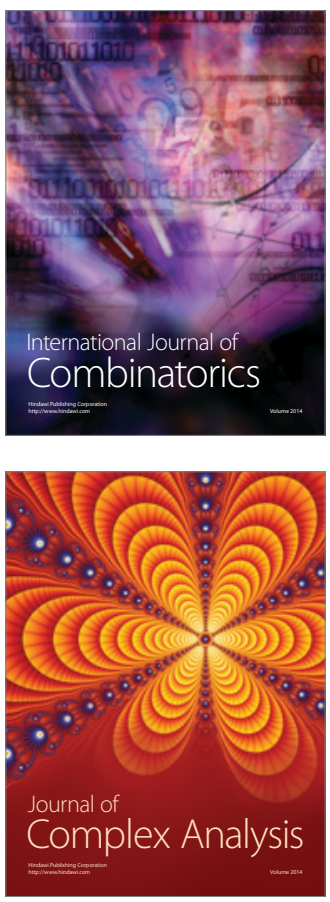

International Journal of

Mathematics and

Mathematical

Sciences
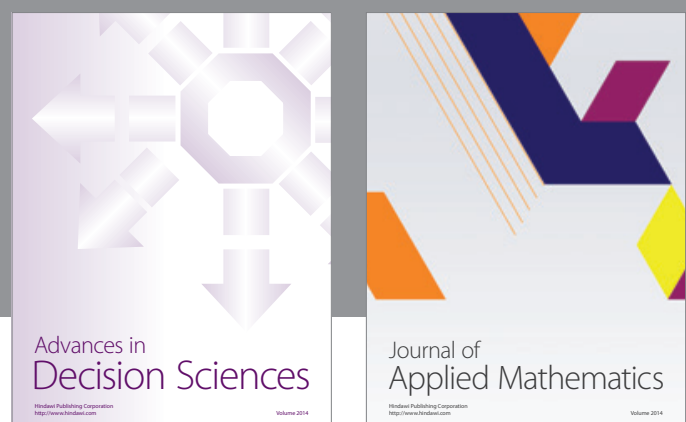

Journal of

Applied Mathematics
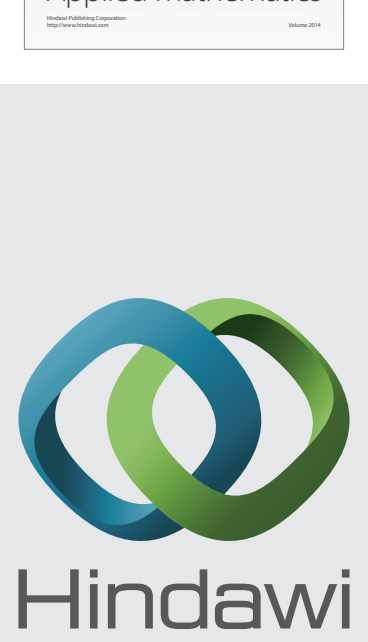

Submit your manuscripts at http://www.hindawi.com
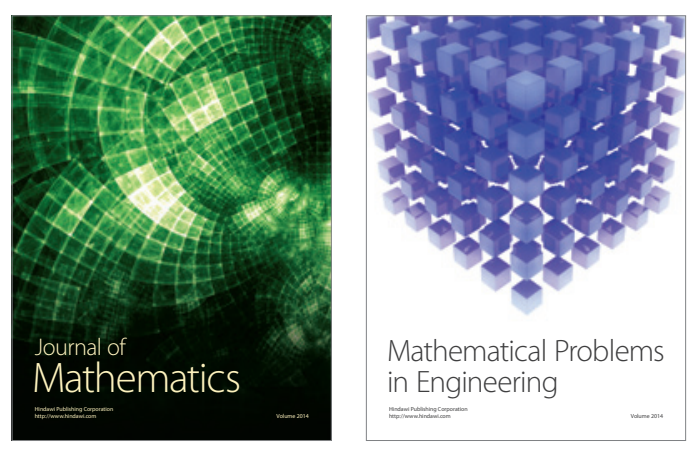

Mathematical Problems in Engineering
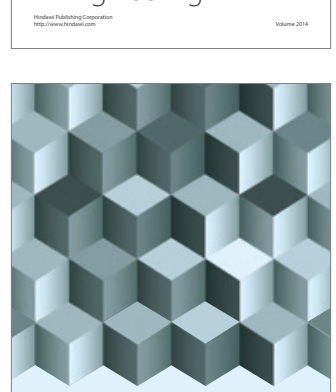

Journal of

Function Spaces
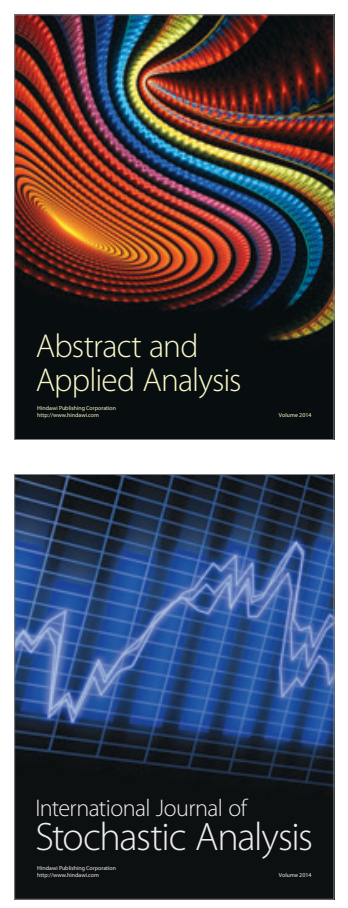

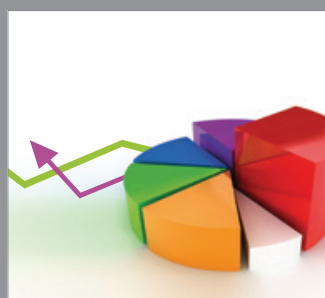

ournal of

Probability and Statistics

Promensencen
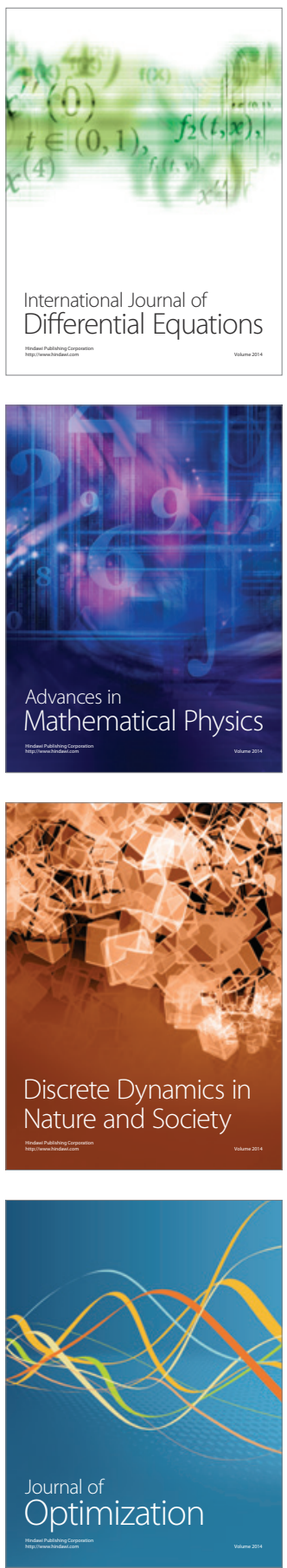